doi:10.13108/2021-13-2-70

\title{
DISCRETE RIEMANN-HILBERT PROBLEM AND INTERPOLATION OF ENTIRE FUNCTIONS
}

\author{
V.YU. NOVOKSHENOV
}

\begin{abstract}
We consider two problems in complex analysis which were developed in Ufa in 1970s years. These are a Riemann-Hilbert problem about jump of a piecewise-analytic function on a contour and a problem of interpolation of entire functions on a countable set in the complex plane. A progress in recent years led to comprehension that they have much common in subject. The first problem arrives as an equivalent of the inverse scattering problem applied for integrating nonlinear differential equations of mathematical physics. The second problem is a natural generalization of Lagrange formula for polynomial with given values on a finite set of points. It is shown that both problems can be united by generalization of the Riemann-Hilbert problem on a case of "discrete contour", where a "jump" of analytic function takes place. This formulation of the discrete matrix Riemann problem can be applied now for various problems of exactly solvable difference equations as well as estimates of spectrum of random matrices. In the paper we show how the discrete matrix Riemann-Hilbert problem provides a way to integrate nonlinear difference equations such as a discrete Painlevé equation. On the other hand, it is shown how assignment of residues to meromorphic matrix functions is effectively reduced to an interpolation problem of entire functions on a countable set in $\mathbb{C}$ with the only accumulation point at infinity. Other application of discrete matrix Riemann-Hilbert problem includes calculation of Fredholm determinants emerging in combinatorics and group representation theory.
\end{abstract}

Keywords: Riemann-Hilbert problem, inverse scattering problem, entire functions, interpolation, canonical product, discrete Painlevé equations, Fredholm determinant, asymptotic expansions.

Mathematics Subject Classification: 30D30, 30E10, 33C10, 33E17, 34M50, 37K60

\section{INTRODUCTION}

Alexey Borisovich Shabat invented the method of the Riemann-Hilbert problem as equivalent of the inverse scattering method in Ufa in 1975 [7, [8]. His idea was based on an adequate description analytic properties of the matrix $\Psi$-function that satisfies the scattering problem

$$
\begin{aligned}
& \frac{d \Psi}{d x}=(i \lambda A+V(x)) \Psi, \quad x \in \mathbb{R}, \\
& \Psi(x, \lambda) \rightarrow \begin{cases}e^{i \lambda A x}, & x \rightarrow+\infty, \\
e^{i \lambda A x} S(\lambda), & x \rightarrow-\infty,\end{cases}
\end{aligned}
$$

V.Yu. Novokshenov, Discrete Riemann-Hilbert problem and interpolation of entire funcTIONS.

(C) Novokshenov V.Yu. 2021.

Submitted March 28, 2021. 
where $\Psi \in \operatorname{Mat}(n, \mathbb{C})$,

$$
\begin{gathered}
A=\operatorname{diag}\left(a_{1}, a_{2}, \ldots, a_{n}\right), \quad a_{1}<a_{2}<\ldots<a_{n}, \\
V(x) \in \operatorname{Mat}(n, \mathbb{R}), \quad V_{j j}=0, \quad V_{j k} \in L_{1}(\mathbb{R}) .
\end{gathered}
$$

The inverse scattering problem (ISP) is to recover the matrix $\Psi(x, \lambda)$, for all $x, \lambda \in \mathbb{R}$ satisfying conditions (1.1) by (1.2) by a given scattering matrix $S(\lambda)$ and a constant matrix $A$. In this way one recovers the potential $V(x)$ in equation (1.1).

More precisely, we introduce a Wiener Banach algebra

$$
\mathcal{W}=\left\{f \mid f(\lambda)=\int_{-\infty}^{\infty} \tilde{f}(x) e^{i \lambda x} d x, \quad \tilde{f} \in L_{1}(\mathbb{R})\right\}
$$

and its two subalgebras $\mathcal{W}_{1}$ and $\mathcal{W}_{2}$

$$
\mathcal{W}_{1}=\left\{f(\lambda)=\int_{0}^{\infty} \tilde{f}(x) e^{i \lambda x} d x,\right\}, \quad \mathcal{W}_{2}=\left\{f(\lambda)=\int_{-\infty}^{0} \tilde{f}(x) e^{i \lambda x} d x,\right\}
$$

with the norm

$$
\|f\|_{\mathcal{W}}=\int_{-\infty}^{\infty}\|\tilde{f}(x)\| d x
$$

It is easy to prove [8] that the scattering matrix $S(\lambda)$ of equation 1.1 with conditions (1) possesses the properties

$$
\begin{gathered}
1-S \in \mathcal{W}, \\
1-\operatorname{det}_{j} S \in \mathcal{W}_{1}, \quad 1-\operatorname{det}_{j} S^{-1} \in \mathcal{W}_{2},
\end{gathered}
$$

where $\operatorname{det}_{j} S$ is the main $j$ th minor of the matrix $S, j=1,2, \ldots, n$. These properties allow to pass from the matrix $S$ to jump matrix $Q$ determining the Riemann-Hilbert problem:

$$
\begin{gathered}
S=N_{1} M_{1}^{-1}=N_{2} M_{2}^{-1}, \\
Q=M_{2} M_{1}^{-1}=N_{2} N_{2}^{-1}, \\
\operatorname{diag} M_{1}=\left\{\operatorname{det}_{1} S, \ldots, \operatorname{det}_{n} S\right\}, \quad \operatorname{diag} N_{2}=\left\{\operatorname{det}_{1} S^{-1}, \ldots, \operatorname{det}_{n} S^{-1}\right\},
\end{gathered}
$$

where $M_{1}, N_{2}$ are upper-triangle matrices and $M_{2}, N_{1}$ are lower-triangle ones.

Theorem 1.1. [7, 8] Assume that the following Riemann-Hilbert problem is solvable:

1) $\Phi_{ \pm}(x, \lambda) \in \operatorname{Mat}(n, \mathbb{C})$ are analytic in the upper $(+)$ and lower (-) half-plane of $\lambda$,

$$
I-\Phi_{+} \in \mathcal{W}_{1}, \quad I-\Phi_{-} \in \mathcal{W}_{2}, \quad \operatorname{det} \Phi_{ \pm}=1,
$$

2) $\Phi_{ \pm}(x, \lambda) \rightarrow I$ as $\lambda \rightarrow \infty, \quad \operatorname{Im} \lambda \gtrless 0$,

3) $\Phi_{-}(x, \lambda)=\Phi_{+}(x, \lambda) Q(x, \lambda), \quad \lambda \in \mathbb{R}$, where $Q(x, \lambda)=e^{-i x \lambda A} Q(\lambda) e^{-i x \lambda A}$,

then the functions

$$
\Psi_{1}(x, \lambda)=\Phi_{+}(x, \lambda+i 0) e^{i x \lambda A}, \quad \Psi_{2}(x, \lambda)=\Phi_{-}(x, \lambda-i 0) e^{i x \lambda A}
$$

satisfy equations (1.1) with the potentials

$$
V(x)=\lim _{\lambda \rightarrow \infty} i \lambda\left[\Phi_{+}, A\right] \Phi_{+}^{-1}(x, \lambda)
$$

and scattering condition (1.2) with the matrix $S$ defined by formulae (1).

In the soliton theory, the $\Psi$-function and the potential $V$ depend as a rule on an additional independent variable $t$, while 1.1 is complemented by one more equation of form

$$
\frac{d \Psi}{d t}=\left(i \lambda^{n} A+\lambda^{n-1} V_{n-1}(x, t)+\ldots+V(x, t)\right) \Psi .
$$

The solvability condition of this equation (1.1) (Lax pair) provides a nonlinear partial differential equation for the function $V(x, t)$, while the scattering problem (1.1), 1.2) plays a role of a "Fourier transform" for finding its solution [2]. 
Theorem 1.1 by A.B. Shabat turned out to be extremely useful for the theory of solitons, just as the Fourier analysis is useful for linear differential equations. In particular, the fact that in the RiemannHilbert problem the variables $x, t$ and the potential $V(x, t)$ appear as parameters, greatly simplified the asymptotic analysis of solutions of the nonlinear equation, which is satisfied by $V(x, t)$. Later the formulation of the Riemann-Hilbert problem was extended to other exactly solvable nonlinear equations, including various evolutionary equations with two spatial variables, difference equations, systems of equations of classical mechanics, etc. At present, the inverse problem method is often formulated only in the form of one or another Riemann-Hilbert problem.

Another large topic had been developing in Ufa in the 1970s, was the theory of entire functions and, in particular, the problem of interpolating these functions in different spaces. In 1976, Alexey Fedorovich Leontiev published his monograph [4], in which he considered various aspects of the theory of Dirichlet series, equations in convolutions and other classical questions of the theory of entire functions. Yet in 1948 A.F. Leontiev was the first to consider the interpolation problem in the space of entire functions of finite nonzero order, which was later called the problem of free interpolation. The notion "free interpolation" is related to the fact that on the values interpolating function, belonging to a given space functions, the smallest restrictions are imposed, and each function from this space should satisfy.

A classical interpolation problem is to find a function $F$ in a given class taking given values $\left\{b_{n}\right\}$ at prescribed points $\left\{a_{n}\right\}$, interpolation nodes:

$$
F\left(a_{n}\right)=b_{n}, \quad n \in \mathbb{N} .
$$

In his work [5], A.F. Leontiev formulated the free interpolation problem as follows: to determine, what conditions should be satisfied by a sequence of different points $\left\{a_{n}\right\}$ in the complex plane so that for each sequence of numbers $\left\{b_{n}\right\}$ satisfying the inequality

$$
\lim _{n \rightarrow \infty} \sup \frac{\ln ^{+} \ln ^{+}\left|b_{n}\right|}{\ln r} \leqslant \rho, \quad \rho>0
$$

in order to construct an entire function $F(z)$ in the class $[\rho, \infty]$ satisfying identities $(1.6)$. The class $[\rho, \infty]$ consists of entire functions possessing normal or minimal type under a given specified order $\rho$.

A function in the class $[\rho, \infty]$ is constructed by means of a generalized Lagrange series

$$
F(z)=\sum_{n=1}^{\infty} \frac{b_{n} \Phi(z) \omega(z)}{\left(z-a_{n}\right) \Phi^{\prime}\left(a_{n}\right) \omega\left(a_{n}\right)}
$$

where $\omega(z)$ is an entire function of order at most $\rho$ and

$$
\Phi(z)=\prod_{n=1}^{\infty}\left(1-\frac{z}{a_{n}}\right) e^{P_{n}(z)}, \quad P_{n}(z)=\frac{z}{a_{n}}+\frac{1}{2}\left(\frac{z}{a_{n}}\right)^{2}+\ldots+\frac{1}{q_{n}}\left(\frac{z}{a_{n}}\right)^{q_{n}}
$$

is a canonical function of sequence $\left\{a_{n}\right\}$ and $q_{n}$ is a sequence of natural numbers ensuring the convergence of series 1.7 in the space $[\rho, \infty])$. A.F. Leontiev proved the following theorem.

Theorem 1.2. [5] Problem (1.6) is solvable in the space $[\rho, \infty], \rho>0$, if and only if the condition

holds.

$$
\lim _{n \rightarrow \infty} \sup \frac{1}{\ln \left|a_{n}\right|} \ln ^{+} \ln \frac{1}{\Phi^{\prime}\left(a_{n}\right)} \leqslant \rho
$$

The two aforementioned problems of complex analysis seemed not to be related one with the other. It seemed so for almost thirty years, till 2000s, when in the works of Alexei Mikhailovich Borodin and Andrey Yuryevich Okunkov on a discrete version of the Riemann-Hilbert problem [9], 10, [11] appeared. On the one hand, like the classical Riemann-Hilbert problem on contour in Theorem 1.1. it was aimed on solving some nonlinear equation. On the other hand, as in Theorem 1.2 , it was very similar to the problem of free interpolation of an entire function on a countable set of nodes.

The specific features of the Riemann-Hilbert problems for the discrete case are to be mentioned. Here the problem of adjoining the boundary values on a continuous contour in the complex plane is replaced by specifying the residues of a meromorphic function on a discrete set of points. In the soliton theory, an analogue of this problem is the recovering of the eigenfunctions by the discrete spectrum 
of a given operator, which is equivalent to solving the Riemann-Hilbert problem with a finite number of zeros det $\Psi_{ \pm}$in the corresponding domains of analyticity. In the discrete case, the singular part of the $\Psi$-function degenerates at countably many points. Below, in Section 2 , this approach will be illustrated by the example of integrating a discrete Painlevé equation of the second type and calculating the Fredholm determinant of one integral operator arising in the theory of random matrices.

In the final Section 3 we discuss the solvability of the discrete matrix Riemann-Hilbert problem. This problem turns out to be equivalent to an interpolation of an entire function on a countable set of nodes. The Lagrange interpolation series (1.7) is modified in such a way as to serve the case of matrix coefficients and the adjoining condition on a countable number of nodes. As an illustration, an interpolation series is calculated for one exact solution of the discrete matrix Riemann-Hilbert problem.

\section{Discrete Riemann-Hilbert PROBlem}

Following works by A.M. Borodin [10], [11, we define a discrete matrix Riemann-Hilbert problem $(\mathrm{DMRH})$ as follows.

Let $\Sigma$ be some countable set of points in the complex plane $\lambda \in \mathbb{C}$ with the only accumulation point at infinity. Let $H(x)$ be a nilpotent matrix function on $\Sigma, H: \Sigma \rightarrow \operatorname{Mat}(N, \mathbb{C}), H^{2}(x)=0$.

We say that a matrix-valued function $Y: \mathbb{C} \backslash \Sigma \rightarrow \operatorname{Mat}(N, \mathbb{C})$ with simple poles at the points $x \in \Sigma$ is a solution of the discrete Riemann-Hilbert problem $(\Sigma, H)$ if the following conditions are satisfied:

$1^{\circ} Y(\lambda)$ is analytic in $\mathbb{C} \backslash \Sigma$ and has simple poles at the points $\Sigma$,

$2^{\circ} \operatorname{Res}_{\lambda=x} Y(\lambda)=\lim _{\lambda \rightarrow x}(Y(\lambda) H(x)), \quad x \in \Sigma$,

$3^{\circ} Y(\lambda) \rightarrow I$ as $\lambda \rightarrow \infty$.

As above, $H(\lambda)$ is called jump matrix.

We note that Condition $3^{\circ}$ means that the function $Y(\lambda)$ has an essential singularity at infinity. Indeed, a function with poles accumulating to infinity cannot have regular asymptotics. In order to the condition to be correct, we require, for example, an uniform asymptotics on a sequence of circumferences $|\lambda|=a_{k}, a_{k} \rightarrow+\infty$. Moreover, we shall assume that there exists a sequence of expanding contours such that the distance from them to the set $\Sigma$ is separated from zero, and we suppose that the solution $Y(\lambda)$ possesses the required asymptotics on these contours.

The issue on existence of solutions to matrix Riemann-Hilbert problem, both the classical one (Theorem 1.1) and the discrete one $1^{\circ}-3^{\circ}$, is rather complicated [12, [13]. We consider it in Section 3. At the same time, the uniqueness of the solutions is proved in a rather simple way.

Theorem 2.1. Under the assumptions of Theorem 1.1, a solution of Riemann-Hilbert problem $\Phi_{ \pm}(x, \lambda)$ is unique.

Proof. Assume that there exist two solutions $\Phi_{ \pm}(x, \lambda)$ and $\chi_{ \pm}(x, \lambda)$. We consider matrix functions $\Phi_{+}(x, \lambda) \chi_{+}^{-1}(x, \lambda)$ and $\Phi_{-}(x, \lambda) \chi_{-}^{-1}(x, \lambda)$. By Condition 1$)$, these functions are analytic in $\lambda$ respectively in the upper and lower half-plane, while on the real axis they coincide by Condition 3). At infinity, they tend to the unit matrix due to Condition 2). Therefore, by the Liouville theorem, they are identically unit and hence $\Phi_{ \pm}(x, \lambda)=\chi_{ \pm}(x, \lambda)$. The proof is complete.

The proof of the uniqueness of a solution to DMRH is a bit more complicated but it is also based on the Liouville problem.

Theorem 2.2. [10] Solution of Riemann-Hilbert problem $Y(\lambda)$ obeying Conditions $1^{\circ}-3^{\circ}$ is unique.

Proof. First we are going to prove that the matrix $Y(\lambda)\left(I+\frac{H(x)}{\lambda-x}\right)$ is analytic in the vicinity of the point $x$. By Condition $1^{\circ}$ we have:

$$
\begin{gathered}
Y(\lambda)=\frac{A(x)}{\lambda-x}+B(x)+O(\lambda-x), \quad \lambda \rightarrow x \\
\operatorname{Res}_{\lambda=x} Y(\lambda)=A(x), \quad Y(\lambda) H(x)=\frac{A(x) H(x)}{\lambda-x}+B(x) H(x)+O(\lambda-x) .
\end{gathered}
$$


Then by Condition $2^{\circ}$, which states $\operatorname{Res}_{\lambda=x} Y(\lambda)=\lim _{\lambda \rightarrow x}(Y(\lambda) H(x))$, we get

$$
A(x) H(x)=0, \quad A(x)=\lim _{\lambda \rightarrow x}(Y(\lambda) H(x))=B(x) H(x) .
$$

Hence, taking into consideration the condition $H^{2}(x)=0$, we obtain

$$
Y(\lambda)\left(I+\frac{H(x)}{\lambda-x}\right)=B(x)+O(1)
$$

and this means the analyticity in the vicinity of $x$.

Assume that $Y_{1}(\lambda)$ and $Y_{2}(\lambda)$ are two different solutions of DMRH $1^{\circ}-3^{\circ}$. Then the matrix

$$
\begin{aligned}
Y_{1}(\lambda) Y_{2}^{-1}(\lambda) & =Y_{1}(\lambda)\left(I+\frac{H(x)}{\lambda-x}\right)\left(I+\frac{H(x)}{\lambda-x}\right)^{-1} Y_{2}^{-1}(\lambda) \\
& =Y_{1}(\lambda)\left(I+\frac{H(x)}{\lambda-x}\right)\left(Y_{2}(\lambda)\left(I+\frac{H(x)}{\lambda-x}\right)\right)^{-1}
\end{aligned}
$$

is analytic in $\mathbb{C}$ since $x$ is an arbitrary point in $\Sigma$.

By Condition $3^{\circ}$, we have $Y_{1}(\lambda) Y_{2}^{-1}(\lambda) \rightarrow I$ as $\lambda \rightarrow \infty$, then by the Liouville theorem this function is identically equal to the unit matrix. This completes the proof.

Now we give some examples of employing DMRH in nonlinear problems of mathematical physics.

We begin with obtaining the Lax pair for a nonlinear difference equation, which can be solved by means of DMRH. As in the continuous case by A.B. Shabat, we need to obtain two linear matrix equations in the variables $\lambda$ and $x$, respectively. The compatibility condition of this Lax pair is the sought nonlinear equation [14, [17.

We denote

$$
\mathbb{Z}^{\prime}=\mathbb{Z}+\frac{1}{2}=\left\{\ldots,-\frac{3}{2},-\frac{1}{2}, \frac{1}{2}, \frac{3}{2}, \ldots\right\}=\mathbb{Z}_{+}^{\prime} \cup \mathbb{Z}_{-}^{\prime},
$$

where $\mathbb{Z}_{+}^{\prime}=\left\{\frac{1}{2}, \frac{3}{2}, \ldots\right\}$ and $\mathbb{Z}_{-}^{\prime}=\left\{\ldots,-\frac{3}{2},-\frac{1}{2}\right\}$.

We consider the construction of Lax pair for problem $1^{\circ}-3^{\circ}$ in a particular case $N=2$ and $\Sigma=\Sigma_{k}$, where

$$
\begin{aligned}
& \Sigma_{k}=\{k, k+1, k+2, \ldots\}, \quad k \in \mathbb{Z}^{\prime},
\end{aligned}
$$

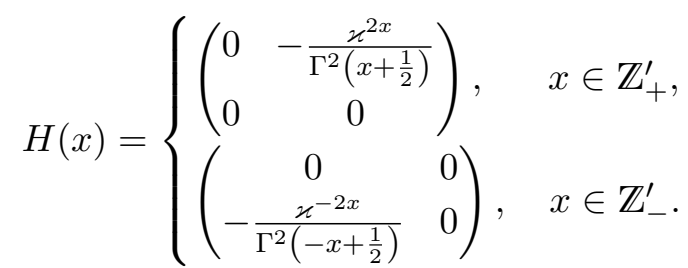

Following [1] and [6], let us prove that for each $n \in \mathbb{Z}_{k}$ there exists a constant nilpotent matrix $A_{n}$,

$$
A_{n}=\left(\begin{array}{cc}
p_{n} & q_{n} \\
r_{n} & -p_{n}
\end{array}\right), \quad p_{n}^{2}=-r_{n} q_{n}
$$

and the functions $a_{n}, b_{n}, a_{n} b_{n}=1$ such that

$$
\begin{gathered}
Y_{n+1}(\lambda)=\left(I+\frac{A_{n}}{\lambda-n}\right) Y_{n}(\lambda), \\
Y_{n}(\lambda-1)\left(\begin{array}{cc}
\varkappa^{-1}\left(\lambda-\frac{1}{2}\right) & 0 \\
0 & \varkappa\left(\lambda-\frac{1}{2}\right)^{-1}
\end{array}\right)=\left(\begin{array}{cc}
\varkappa^{-1}\left(\lambda-\frac{1}{2}-p_{n}\right) & a_{n} \\
-b_{n} & 0
\end{array}\right) Y_{n+1}(\lambda) .
\end{gathered}
$$

Indeed, since $H$ is independent of $n$, we see that $Y_{n}(\lambda)$ and $Y_{n+1}(\lambda)$ satisfy the same jump condition on $\Sigma_{n}$. However, $Y_{n+1}$ has an additional pole at the point $\{n\}=\Sigma_{n+1} \backslash \Sigma_{n}$. Therefore, the quotient $Y_{n+1} Y_{n}^{-1}$ possesses a single pole at the point $\lambda=n$. Denoting the residue at this point by $A_{n}$, we conclude that the function

$$
Y_{n+1}(\lambda) Y_{n}^{-1}(\lambda)-\frac{A_{n}}{\lambda-n}
$$


is entire. Calculating the asymptotics in the vicinity of $\lambda=\infty$, by the Liouville theorem we obtain that this function is identically equal to $I$ and this proves the first equation.

Next, it follows from $\operatorname{det} Y_{n} \equiv \operatorname{det} Y_{n+1} \equiv 1$ that $\operatorname{det}\left(I+A_{n} /(\lambda-n)\right) \equiv 1$. This implies that $A_{n}$ is nilpotent.

The derivation of equation (2.7) is a bit more complicated. It follows from Condition $3^{\circ}$ that

$$
Y_{n}(\lambda)=I+\left(\begin{array}{cc}
\alpha_{n} & \beta_{n} \\
\gamma_{n} & \delta_{n}
\end{array}\right) \lambda^{-1}+O\left(\lambda^{-2}\right), \quad \lambda \rightarrow \infty,
$$

with some constants $\alpha_{n}, \ldots, \delta_{n}$.

We divide both sides of equation (2.7) from the left by the matrix $Y_{n+1}(\lambda)$ and let us prove that its left hand side a polynomial in $\lambda$.

By (2.8), the asymptotics as $\lambda \rightarrow \infty$ is of the form

$$
\begin{gathered}
\left.\left(I+\left(\begin{array}{cc}
\alpha_{n} & \beta_{n} \\
\gamma_{n} & \delta_{n}
\end{array}\right) \lambda^{-1}\right)\left(\begin{array}{cc}
\varkappa^{-1}\left(\lambda-\frac{1}{2}\right) & 0 \\
0 & 0
\end{array}\right)\left(I-\left(\begin{array}{cc}
\alpha_{n+1} & \beta_{n+1} \\
\gamma_{n+1} & \delta_{n+1}
\end{array}\right) \lambda^{-1}\right)\right)+O\left(\lambda^{-1}\right) \\
=\varkappa^{-1}\left(\begin{array}{cc}
\lambda-\frac{1}{2}+\alpha_{n}-\alpha_{n+1} & -\beta_{n+1} \\
\gamma_{n} & 0
\end{array}\right)+O\left(\lambda^{-1}\right) .
\end{gathered}
$$

We denote $a_{n}=-\varkappa^{-1} \beta_{n+1}, b_{n}=-\varkappa^{-1} \gamma_{n}, c_{n}=\alpha_{n+1}-\alpha_{n}$. Then it follows from the Liouville theorem that expression (2) is equal to

$$
\left(\begin{array}{cc}
\varkappa^{-1}\left(\lambda-\frac{1}{2}-c_{n}\right) & a_{n} \\
-b_{n} & 0
\end{array}\right) .
$$

In conclusion, let us prove that $c_{n}=p_{n}$ and $a_{n} b_{n}=1$. The second identity is implied by the fact that the determinant $Y_{n}(\lambda)$ is equal to 1 . In order to prove that $c_{n}=p_{n}$, we substitute 2.6) in the just proven relation $(2.7)$. We obtain:

$Y_{n}(\lambda-1)\left(\begin{array}{cc}\varkappa^{-1}\left(\lambda-\frac{1}{2}\right) & 0 \\ 0 & \varkappa\left(\lambda-\frac{1}{2}\right)^{-1}\end{array}\right)=\left(\begin{array}{cc}\varkappa^{-1}\left(\lambda-\frac{1}{2}-c_{n}\right) & a_{n} \\ -b_{n} & 0\end{array}\right)\left(I+(\lambda-n)^{-1}\left(\begin{array}{cc}p_{n} & q_{n} \\ r_{n} & -p_{n}\end{array}\right)\right) Y_{n}(\lambda)$.

Comparing the asymptotics of the matrix entries $(\cdot)_{11}$ in this identity, we see that $c_{n}=p_{n}$. Thus, we have proved the validity of the equations in Lax pair 2.6 and 2.7).

Theorem 2.3. The compatibility condition of the equations in Lax pair (2.6) and (2.7) is the discrete Painlevé equation of second type (dPII)

$$
v_{n+1}+v_{n-1}=\frac{\left(n+\frac{1}{2}\right) v_{n}}{\varkappa\left(v_{n}^{2}-1\right)},
$$

where $v_{n}^{2}=\varkappa^{-1} a_{n} r_{n}$.

Proof. Shifting $\lambda$ by 1 in (2.7) and substituting the right side of 2.7) into that of (2.6), we obtain:

$$
Y_{n+1}(\lambda)=\left(I+\frac{A_{n}}{\lambda-n}\right)\left(\begin{array}{cc}
\varkappa^{-1}\left(\lambda+\frac{1}{2}-p_{n}\right) & a_{n} \\
-b_{n} & 0
\end{array}\right) Y_{n+1}(\lambda+1)\left(\begin{array}{cc}
\varkappa\left(\lambda+\frac{1}{2}\right)^{-1} & 0 \\
0 & \varkappa^{-1}\left(\lambda+\frac{1}{2}\right)
\end{array}\right) .
$$

On the other hand, shifting $n$ and $\lambda$ by 1 in (2.6) and (2.7) and substituting the right hand side of (2.6) into that of (2.7), we obtain

$$
Y_{n+1}(\lambda)=\left(\begin{array}{cc}
\varkappa^{-1}\left(\lambda+\frac{1}{2}-p_{n+1}\right) & a_{n+1} \\
-b_{n+1} & 0
\end{array}\right)\left(I+\frac{A_{n+1}}{\lambda-n}\right) Y_{n+1}(\lambda+1)\left(\begin{array}{cc}
\varkappa\left(\lambda+\frac{1}{2}\right)^{-1} & 0 \\
0 & \varkappa^{-1}\left(\lambda+\frac{1}{2}\right)
\end{array}\right) .
$$

Comparing these two relations, we obtain the compatibility condition for Lax pair (2.6), 2.7):

$$
\left(I+\frac{A_{n}}{\lambda-n}\right)\left(\begin{array}{cc}
\varkappa^{-1}\left(\lambda+\frac{1}{2}-p_{n}\right) & a_{n} \\
-b_{n} & 0
\end{array}\right)=\left(\begin{array}{cc}
\varkappa^{-1}\left(\lambda+\frac{1}{2}-p_{n+1}\right) & a_{n+1} \\
-b_{n+1} & 0
\end{array}\right)\left(I+\frac{A_{n+1}}{\lambda-n}\right) .
$$

By matrix equation (2.11) we can easily obtain scalar equations for the variables $p_{n}$ and $r_{n}$. Namely, calculating the asymptotics of the elements $(\cdot)_{12}$ and $(\cdot)_{21}$ in identity (2.11) as $\lambda \rightarrow \infty$, we arrive at the relations:

$$
\left\{\begin{array}{l}
a_{n}=a_{n+1}+\varkappa^{-1} q_{n+1}, \quad b_{n}=b_{n+1}+\varkappa^{-1} r_{n}, \\
a_{n} r_{n}=-b_{n+1} q_{n+1} .
\end{array}\right.
$$


The residues in a simple pole at $\lambda=n$ in identity 2.11) read as

$$
\left(\begin{array}{cc}
p_{n} & q_{n} \\
r_{n} & -p_{n}
\end{array}\right)\left(\begin{array}{cc}
\varkappa^{-1}\left(n+\frac{1}{2}-p_{n}\right) & a_{n} \\
-b_{n} & 0
\end{array}\right)=\left(\begin{array}{cc}
\varkappa^{-1}\left(n+\frac{1}{2}-p_{n+1}\right) & a_{n+1} \\
-b_{n+1} & 0
\end{array}\right)\left(\begin{array}{cc}
p_{n+1} & q_{n+1} \\
r_{n+1} & -p_{n+1}
\end{array}\right) .
$$

The entry $(\cdot)_{22}$ in this identity coincide with identity $(2.12)$, while the entry $(\cdot)_{12}$ gives

$$
a_{n} p_{n}=\varkappa^{-1}\left(\lambda+\frac{1}{2}-p_{n+1}\right) q_{n+1}-a_{n+1} p_{n+1} .
$$

Substituting both sides by $b_{n+1}$ and recalling that $a_{n+1} b_{n+1}=1$, we obtain:

$$
b_{n+1} a_{n} p_{n}=-\varkappa^{-1}\left(\lambda+\frac{1}{2}-p_{n+1}\right) a_{n} r_{n}-p_{n+1} .
$$

We denote

$$
s_{n}=a_{n} r_{n},
$$

then, multiplying the first relation in 2.12 by $b_{n+1}$, we see that $a_{n} b_{n+1}=1-\varkappa^{-1} s_{n}$. Substituting this expression into (2.13), we obtain

$$
\left(p_{n}+p_{n+1}\right)\left(s_{n}-\varkappa\right)=\left(n+\frac{1}{2}\right) s_{n},
$$

Employing the nilpotent property of the matrix $A_{n}$ (2.5), we find:

$$
p_{n+1}^{2}=-q_{n+1} r_{n+1}=\left(-b_{n+1} q_{n+1}\right)\left(a_{n+1} r_{n+1}\right)=s_{n} s_{n+1} .
$$

Thus, for each $n \in \mathbb{Z}_{k}$ we obtain a system of scalar equations

$$
\left\{\begin{array}{l}
\left(p_{n}+p_{n+1}\right)\left(s_{n}-\varkappa\right)=\left(n+\frac{1}{2}\right) s_{n}, \\
p_{n+1}^{2}=s_{n} s_{n+1} .
\end{array}\right.
$$

It is easy to exclude the variable $p_{n}$ from this system, namely, letting

$$
s_{n}=\varkappa v_{n}^{2},
$$

we obtain scalar difference equation 2.10.

We note that in works [16], [18, the discrete Painlevé equation dPII was derived by symmetry arguing without using the technique of DMRH.

As another example of applying DMRH, the calculation of Fredholm determinants for integrals operators serves. There determinants are related with the group representation theory and problems in combinatorics. An example of the mentioned operator is an integral operator with a Bessel kernel [13], [19]

$$
Q(x, y)=\varkappa \frac{J_{x-\frac{1}{2}}(2 \varkappa) J_{y+\frac{1}{2}}(2 \varkappa)-J_{y-\frac{1}{2}}(2 \varkappa) J_{x+\frac{1}{2}}(2 \varkappa)}{x-y},
$$

where $\varkappa$ is a parameter and $J_{\nu}$ is the Bessel $J$-function.

We let $x, y \in \mathbb{Z}^{\prime}=\left\{\ldots,-\frac{3}{2},-\frac{1}{2}, \frac{1}{2}, \frac{3}{2}, \ldots\right\}$ and then we denote

$$
\Sigma_{k}=\{k, k+1, k+2 \ldots\}, \quad k \in \mathbb{Z}^{\prime} .
$$

We define $Q_{k}$ as the restriction of the operator $Q$ on $\ell_{2}\left(\Sigma_{k}\right)$

$$
Q_{k} f(x)=\sum_{y=k}^{\infty} Q(x, y) f(y), \quad f \in \ell_{2}\left(\Sigma_{k}\right) .
$$

The operator $Q_{k}$ is nuclear and positive definite [11] and this is why its Fredholm determinant is well-defined:

$$
D_{k}=\operatorname{det}\left(1-Q_{k}\right), \quad D_{k} \neq 0
$$

We let $R_{s}=Q_{k}\left(1-Q_{k}\right)^{-1}$. This operator can be expressed by the solution to the following DMRH [11:

(a) $Y(\lambda)$ is analytic in $\mathbb{C} \backslash \Sigma$ and has simple poles at the points $\Sigma$,

(b) $\operatorname{Res}_{\lambda=x} Y(\lambda)=\lim _{\lambda \rightarrow x}(Y(\lambda) H(x)), \quad x \in \Sigma_{k}, \quad H^{2}(x)=0$, 
(c) $\operatorname{det} Y(\lambda) \equiv 1$,

where the jump matrix $H$ is determined by formula (2.4). We observe that problem $1^{\circ}-3^{\circ}$ differs from problem (a)-(c) the normalization condition (c), that is, we do not suppose the boundedness of the solution $Y(\lambda)$ at infinity like in $3^{\circ}$.

Assume that there exists the solution of DMRH (a)-(c) of the form

$$
Y(\lambda)=\left(\begin{array}{cc}
\phi & \hat{\phi} \\
\psi & \hat{\psi}
\end{array}\right)
$$

then the kernel of the operator $R_{k}$ is represented as

$$
R_{k}(x, y)= \begin{cases}\frac{\phi(x) \psi(y)-\phi(y) \psi(x)}{x-y}, & x \neq y, \\ \psi(x) \hat{\psi}(x)-\phi(x) \hat{\phi}(x), & x=y .\end{cases}
$$

It turns out [11] that DNRP (a)-(c) admits an explicit solution

$$
Y(\lambda)=\sqrt{\varkappa}\left(\begin{array}{cc}
J_{\lambda-\frac{1}{2}}(2 \varkappa) & J_{-\lambda+\frac{1}{2}}(2 \varkappa) \\
-J_{\lambda+\frac{1}{2}}(2 \varkappa) & J_{-\lambda-\frac{1}{2}}(2 \varkappa)
\end{array}\right)\left(\begin{array}{cc}
\varkappa^{-\lambda} \Gamma\left(\lambda+\frac{1}{2}\right) & 0 \\
0 & \varkappa^{\lambda} \Gamma\left(-\lambda+\frac{1}{2}\right)
\end{array}\right),
$$

and

$$
1+R_{k}=\frac{\operatorname{det}\left(1-Q_{k+1}\right)}{\operatorname{det}\left(1-Q_{k}\right)}=\frac{D_{k+1}}{D_{k}} .
$$

Theorem 2.4. 9] Let $k \in \mathbb{Z}^{\prime}$ and $v_{k}$ be the solution of discrete Painlevé equation 2.10) with the initial conditions

$$
v_{-\frac{1}{2}}=-1, \quad v_{\frac{1}{2}}=\frac{I_{1}(2 \varkappa)}{I_{0}(2 \varkappa)},
$$

where $I_{0}$ and $I_{1}$ are the Bessel I-functions. Then for all $k \geq \frac{1}{2}$ the relation holds

$$
v_{k}^{2}=1-\frac{D_{k} D_{k+2}}{D_{k+1}^{2}} \text {. }
$$

\section{EXISTENCE OF SOLUTION TO DRMP AND INTERPOLATION PROBLEM}

In view of the applications of DRMP mentioned in Section 2, let us simplify the problem on finding a meromorphic problem satisfying Conditions $1^{\circ}-3^{\circ}$. We consider the set of simple poles $x_{n} \in \Sigma$ distributed in $\mathbb{C}$ with the conditions

$$
0<\operatorname{Re} x_{1} \leqslant \operatorname{Re} x_{2} \leqslant \ldots, \quad x_{n}=\frac{n+\alpha}{\sigma}+O\left(n^{-1}\right), \quad n \rightarrow \infty,
$$

and $x_{0}=0$ and $x_{-n}=-x_{n}$.

We define a canonical product as

$$
\Phi(\lambda)=\prod_{n=1}^{\infty}\left(1-\frac{\lambda^{2}}{x_{n}^{2}}\right)
$$

with the growth indicatrix

$$
h(\theta)=\varlimsup_{r \rightarrow \infty} \frac{\ln \left|\Phi\left(r e^{i \theta}\right)\right|}{r}=\pi \sigma|\sin \theta| .
$$

Conditions (3.1) of course satisfy Theorem 1.2 on interpolation nodes since the limit mentioned in this theorem vanishes as $a_{n}=x_{n}$. This is there exists an interpolation of an entire function $F(\lambda)$ with nodes at the points $x_{n}$. However, in the present case we can apply a simpler theorem [3, Ch. II, Thm. 2.6.5 ].

Theorem 3.1. Let $F(\lambda)$ be an entire function obeying the condition

$$
|F(\mu+i \nu)| \leqslant|\mu|^{\alpha+1} \delta(|\mu|) e^{(\pi \sigma-\epsilon)|\nu|}, \quad \epsilon \rightarrow 0, \quad \delta(\mu) \rightarrow 0, \quad \mu \rightarrow \infty,
$$

and the sequence $x_{n}$ satisfies condition (3.1). Then

$$
F(\lambda)=\sum_{n=-\infty}^{\infty} \frac{F\left(x_{n}\right)}{\Phi^{\prime}\left(x_{n}\right)} \frac{\Phi(\lambda)}{\lambda-x_{n}} \text {. }
$$


We are going to employ Theorem 3.1 for constructing the solution of DMRH by a given jump matrix.

Assume that the nodes $x_{n}$ of solution of DMRH $1^{\circ}-3^{\circ}$ satisfy conditions (3.1) and let us define the canonical product by formula $(3.2)$.

Let the entries of the matrices $B(\lambda)$ and $H(\lambda)$ be entire functions and satisfy estimates $(3.3)$ and

$$
\begin{gathered}
\left|B_{i j}(\lambda)\right| \leqslant|\lambda|^{\alpha+1} \delta(|\lambda|) e^{(\pi \sigma-\epsilon)|\operatorname{Im} \lambda|} \\
\left|H_{i j}(\lambda)\right| \leqslant|\lambda|^{\alpha+1} \delta(|\lambda|) e^{(\pi \sigma-\epsilon)|\operatorname{Im} \lambda|}, \quad \delta(|\lambda|) \rightarrow 0, \quad \lambda \rightarrow \infty .
\end{gathered}
$$

By Theorem 3.1, there exists a solution of the interpolation problem

$$
Y(\lambda)=\sum_{n=-\infty}^{\infty}\left(\frac{A\left(x_{n}\right)}{\lambda-x_{n}}+B\left(x_{n}\right)\right) \frac{\Phi(\lambda)}{\Phi^{\prime}\left(x_{n}\right)\left(\lambda-x_{n}\right)},
$$

where the growth order of $A(x)$ is at most $\pi \sigma$.

It is obvious that $Y(\lambda)$ is a meromorphic matrix having simple poles at the nodes $x_{n}=x$ and its Laurent series is

$$
Y(\lambda)=\frac{A(x)}{\lambda-x}+\left(A(x) \frac{\Phi^{\prime \prime}(x)}{2 \Phi^{\prime}(x)}+B(x)\right)+O(\lambda-x), \quad \lambda \rightarrow x .
$$

This expansion implies jump condition $2^{\circ}$ in the form

$$
\operatorname{Res}_{\lambda=x} Y(\lambda)=A(x)=\lim _{\lambda \rightarrow x} Y(\lambda) H(\lambda)=\lim _{\lambda \rightarrow x}\left(A(x) \frac{\Phi^{\prime \prime}(x)}{2 \Phi^{\prime}(x)}+B(x)\right) H(\lambda) .
$$

In order to fit this identity with (2.1), we apply the niplotent property of the matrix $H(\lambda)$ :

$$
A(x)(I-\phi H(x))=B(x) H(x), \quad(I-\phi H(x))(I+\phi H(x))=I, \quad \phi=\frac{\Phi^{\prime \prime}(x)}{2 \Phi^{\prime}(x)} .
$$

We multiply the first identity from the right by $(I+\phi H(x))$ and apply again the nilpotent property of $H(x)$. Then we obtain:

$$
A(x)=B(x) H(x) .
$$

Thus, we have proved the following theorem.

Theorem 3.2. There exists a solution $Y(\lambda)$ of DMRH $1^{\circ}, 2^{\circ}$ (without normalization condition $3^{\circ}$ ) under the distribution of jump nodes (3.1) in the class of entire functions $Y(\lambda) \Phi(\lambda)$ of a finite order.

As it has been mentioned in Theorem 2.2, the solution of DMRH is unique if all three conditions $1^{\circ}-3^{\circ}$ are satisfied. This is why we need to find a way of achieving the normalization condition $3^{\circ}$ at infinity. For the sake of simplicity, we replace normalization condition $3^{\circ}$ by condition (c) $\operatorname{det} Y(\lambda)=1$, which is present in DRMP (a)-(c) . In order to do this, we again employ the nilpotent property of the leading terms in Laurent series (3.4).

According (3.4) and (3.5), at each node $\lambda=x$, the Laurent series reads as

$$
Y(\lambda)=\left(\frac{A(x)}{\lambda-x}+B(x)+O(\lambda-x)\right)=B(x)\left(\frac{H(x)}{\lambda-x}+I+O(\lambda-x)\right) .
$$

Since $H^{2}(x)=0$, we have:

$$
\left(\frac{H(x)}{\lambda-x}+I\right)\left(-\frac{H(x)}{\lambda-x}+I\right)=I
$$

that is, the matrix $I+H(x) /(\lambda-x)$ is non-degenerate and $\operatorname{det} Y(\lambda)=\operatorname{det} B(x)$ as $\lambda=x$.

We divide both sides of formula (3.4) by the scalar factor $\Phi(\lambda)$, which is canonical product (3.2). This does not spoil the convergence of the series and it will be still a meromorphic function with poles at the points $\lambda=x_{n}$

$$
\tilde{Y}(\lambda)=\sum_{n=-\infty}^{\infty} \frac{A\left(x_{n}\right)+B\left(x_{n}\right)\left(\lambda-x_{n}\right)}{\Phi^{\prime}\left(x_{n}\right)\left(\lambda-x_{n}\right)} .
$$


Then this function is bounded at infinity:

$$
\operatorname{det} \tilde{Y}(\lambda)=\operatorname{det} \sum_{n=-\infty}^{\infty} \frac{B\left(x_{n}\right)}{\Phi^{\prime}\left(x_{n}\right)}, \quad \lambda \rightarrow \infty
$$

Condition (b) becomes the system of equations

$$
A\left(x_{n}\right)=\Phi^{\prime}\left(x_{n}\right) \sum_{k \neq n} \frac{B\left(x_{k}\right) H\left(x_{n}\right)}{\Phi^{\prime}\left(x_{k}\right)\left(x_{k}-x_{n}\right)}, \quad n \in \mathbb{Z} .
$$

Thus, the solvability of DRMP (a)-(c) is implied by the solvability the solvability of system of equations (3.7) and (3.8) for the matrices $A\left(x_{n}\right)$ and $B\left(x_{n}\right)$.

As an example we consider the application of interpolation series $(3.6)$ for finding an exact solution 2.16) of DMRH (a)-(c):

$$
Y(\lambda)=\sqrt{\varkappa}\left(\begin{array}{cc}
J_{\lambda-\frac{1}{2}}(2 \varkappa) & J_{-\lambda+\frac{1}{2}}(2 \varkappa) \\
-J_{\lambda+\frac{1}{2}}(2 \varkappa) & J_{-\lambda-\frac{1}{2}}(2 \varkappa)
\end{array}\right)\left(\begin{array}{cc}
\varkappa^{-\lambda} \Gamma\left(\lambda+\frac{1}{2}\right) & 0 \\
0 & \varkappa^{\lambda} \Gamma\left(-\lambda+\frac{1}{2}\right)
\end{array}\right) .
$$

We note that normalization condition $(\mathrm{c}) \operatorname{det} Y(\lambda)=1$ is implied by the known formulae for the Gamma function and Bessel functions [1]

$$
\begin{gathered}
J_{\lambda-\frac{1}{2}}(2 \varkappa) J_{-\lambda-\frac{1}{2}}(2 \varkappa)+J_{-\lambda+\frac{1}{2}}(2 \varkappa) J_{\lambda+\frac{1}{2}}(2 \varkappa)=\frac{\cos \pi \lambda}{\pi \varkappa}, \\
\Gamma\left(\lambda+\frac{1}{2}\right) \Gamma\left(-\lambda+\frac{1}{2}\right)=\frac{\pi}{\cos \pi \lambda} .
\end{gathered}
$$

Theorem 3.3. Let the matrix $Y(\lambda)$ be a solution of $D M R H(a)-(c)$ given by formula (2.16). Then the entries of the matrix are represented by the interpolation series with nodes $x_{n}=n, n \in \mathbb{Z}$

$$
\begin{aligned}
& Y_{11}(\lambda)=\left(\lambda-\frac{1}{2}\right) \sum_{n=0}^{\infty} \frac{\varkappa^{n}}{n !} \frac{J_{n}(2 \varkappa)}{n+\lambda-\frac{1}{2}}, \\
& Y_{12}(\lambda)=-\left(\lambda+\frac{1}{2}\right) \sum_{n=0}^{\infty} \frac{\varkappa^{n}}{n !} \frac{J_{n}(2 \varkappa)}{n-\lambda-\frac{1}{2}} .
\end{aligned}
$$

The matrix entries $Y_{21}$ and $Y_{22}$ satisfy similar representation.

Proof. According to (2.16), the matrix entries are of the form

$$
Y_{11}(\lambda)=\varkappa^{-\lambda+\frac{1}{2}} \Gamma\left(\lambda+\frac{1}{2}\right) J_{\lambda-\frac{1}{2}}(2 \varkappa), \quad Y_{12}(\lambda)=\varkappa^{\lambda+\frac{1}{2}} \Gamma\left(-\lambda+\frac{1}{2}\right) J_{-\lambda-\frac{1}{2}}(2 \varkappa) .
$$

We note that in problem (a)-(c), the jump condition is given at the points $x \in \mathbb{Z}^{\prime}$, that is, $\lambda=x$ corresponds to half-integer values, $\lambda \pm \frac{1}{2}=n, n \in \mathbb{Z}$.

We employ a known Neumann series over Bessel functions with an integer index [1, Ch. 7.15, Eq. (10)]:

$$
\Gamma(\zeta-\mu) J_{\zeta}(2 \varkappa)=\Gamma(\mu+1) \sum_{n=0}^{\infty}\left(\frac{\Gamma(\zeta-\mu+n)}{\Gamma(\zeta+n+1) n !} \varkappa^{\zeta-\mu+n} J_{n+\mu}(2 \varkappa) .\right.
$$

Letting $\mu=0$ and employing the relation $\Gamma(\zeta+n+1)=(\zeta+n) \Gamma(\zeta+n)$, the latter series can be rewritten as

$$
\Gamma(\zeta) J_{\zeta}(2 \varkappa)=\varkappa^{\zeta} \sum_{n=0}^{\infty} \frac{\varkappa^{n}}{n !} \frac{J_{n}(2 \varkappa)}{\zeta+n} .
$$

Passing to the variable $\lambda-\frac{1}{2}=\zeta$ and employing once again the formula $\Gamma\left(\lambda+\frac{1}{2}\right)=\left(\lambda-\frac{1}{2}\right) \Gamma\left(\lambda-\frac{1}{2}\right)$, we arrive at the first formula in 3.9 . The second formula in $(3.9)$ is obtained by the change $\lambda+\frac{1}{2}=\zeta$. The proof is complete. 


\section{BIBLIOGRAPHY}

1. A. Erdélyi, W. Magnus, F. Oberhettinger, F.G. Tricomi. Higher transcendental functions. Vol. II. McGraw-Hill Book Co., New York (1953).

2. S. Novikov, S.V. Manakov, L.P. Pitaevskii, V.E. Zakharov. Theory of solitons. The inverse scattering method. Nauka, Moscow (1980). [Plenum Publ. Corp. New York (1984).]

3. I.I. Ibragimov. Interpolation methods for functions and some of their applications. Nauka, Moscow (1971). (in Russian).

4. A.F. Leontiev. Exponential series. Nauka, Moscow (1976). (in Russian).

5. A.F. Leont'ev. On interpolation in class of entire functions of finite order // Dokl. AN SSSR. 61:5, 785-787 (1948). (in Russian).

6. V.Yu. Novokshenov. Discrete integrable equations and special functions // Ufimskij Matem. Zhurn. 9:3, 119-131 (2017). [Ufa Math. J. 9:3, 118-130 (2017).]

7. A.B. Shabat. Inverse-scattering problem for a system of differential equations // Funkts. Anal. Pril. 9:3, 75-78 (1975). [Funct. Anal. Appl. 9:3, 244-247 (1975).]

8. A.B. Shabat. An inverse scattering problem // Diff. Uravn. 15:10, 1824-1834 (1979). [Diff. Equats. 15:10, 1299-1307 (1980).]

9. A. Borodin, A. Okounkov. A Fredholm determinant formula for Toeplitz determinants // Integral Equations Operator Theory. 37:4, 386-396 (2000).

10. A. Borodin. Discrete gap probabilities and discrete Painlevé equations // Duke Math. J. 117:3, 1-54 (2003).

11. A. Borodin. Isomonodromy transformations of linear systems of difference equations // Ann. Math. 160:3, 1141-1182 (2004).

12. K. Clancey, I. Gohberg. Factorization of matrix functions and singular integral operators // in "Operator Theory: Advances and Applications". 3, Birkhauser Verlag, Basel (1981).

13. P. Deift. Orthogonal polynomials and random matrices: A Riemann-Hilbert approach // Courant Lecture Notes, New York Univ. (1999).

14. A.S. Fokas, A.R. Its, A.A. Kapaev, V.Yu. Novokshenov. Painlevé Transcendents. The RiemannHilbert Approach // in "Mathematical Surveys and Monograph". 128. Amer. Math. Soc., Providence, Rhode Island (2006).

15. F.D. Gakhov. Boundary value problems. Dover Publications, New York (1990).

16. B. Grammaticos, F.W. Nijhof, A. Ramani. Discrete Painlevé equations // in "The Painlevé property". CRM Ser. Math. Phys. Springer, New York, 413-516 (1999).

17. A.R. Its. The Riemann-Hilbert Problem and Integrable Systems // Notic. Amer. Math. Soc. 50:11, 1389-1400 (2003).

18. H. Sakai. Rational Surfaces Associated with Affine Root Systems and Geometry of the Painlevé Equations // Comm. Math. Phys. 220:1, 165-229 (2001).

19. C.A. Tracy, H. Widom. Random unitary matrices, permutations and Painlevé // Comm. Math. Phys. 207:3, 665-685 (1999).

Viktor Yurievich Novokshenov,

Institute of Mathematics,

Ufa Federal Research Center, RAS,

Chernyshevky str. 112,

450008, Ufa, Russia

E-mail: novik53@mail.ru 